LE OMBRE DEI RAGGI X STUDIATE CON LA FOTOGRAFIA, di li.MHIIO VII.I.ARI.

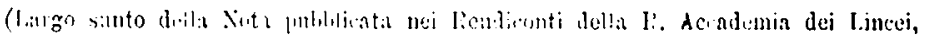
‥1. $i^{n}$, f.r. $.11,1^{0}=(111.159 .0)$.

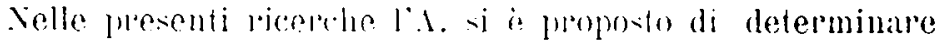

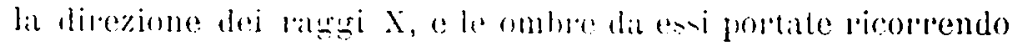
alle immatrini fotonglialiche dat exi pindotte.

lo rildiazoni emene dit un grande focus sferico renivano

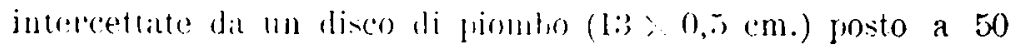
cm. di distanzal dal tuho stemo, l’ombla del quale si proiettara

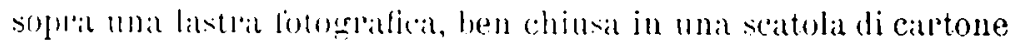
e postal a 4,5 con. di distanzal dal disco di piombo. A megrlio apprezara la di ferenzal di tono delle varie parti della fotoglafia sulla listla sensibile era posta una croce di piombo.

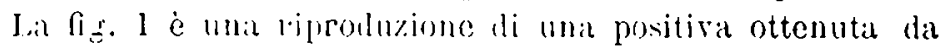

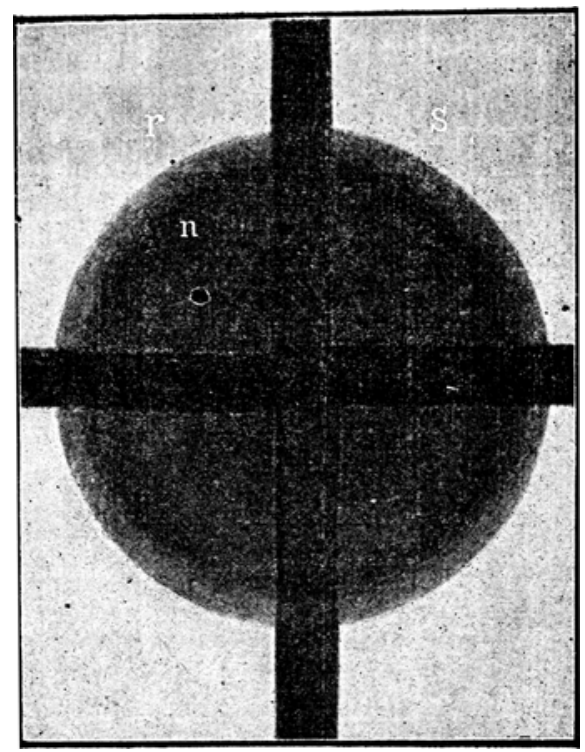

$\mathrm{Fig}^{\circ}$ l.

ma di queste listre che ela stintir esposta da 40 a 50 minuti all'azione del focus. 
In essa si rele un ombra circolatre del disco, assat oscomal, terminata da una zona lallgat da ż all 8 mm., sensibilmente piu chiara, che finisce all esterno a taglion netto e bruscamente,

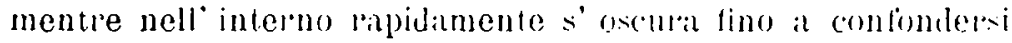
con la tinta piil nera dell ombra. Per fuesto molo brusco di terminarei della \%ona chiara all estermo e per trovarsi come nell' interno dell' ombra, I'A., altralrolta, l'attribui ad mua specie di flessione degrli $\mathrm{X}$, meglio che aul una rera penombra '). Alcune rolte, internamente alla ronal chianal, se ne scol're una piu oscura della tinta frenerale dellat ligurar ed a contorni irregolari ed incerti come, alla moglio, e indicato nella figlual. In questa redesi, inoltre, risultare in osculo, o meglio alla periferia che al centro, l’ombrat dellar croce di fiombo; la quale spicca sui quattro settori, lorse pia chiari allat perileria che al centro. Questa tinta chiara dei setori non fui dipendere dalla trasparenza del disco perché questo è piu grosso della croce, la quale risulti affat to opacal Ne puis dipendere da una specie di flusrescenza cho, scomblo alcuni autori, destata sul fondo della scatola nelle regioni direttamente colpite dagli $X$, si diffonderebbe all intorno sulla lastra. I)ilatti se co. testa diffusione vi fosse dalla pegione $r$, per es., al ricino settore $n$ se ne dovrebbe rerificare una liu enererica di $\gamma$ ed s, sotto l'estremo " della croce, che dovebhe, per conseguenzal, riuscire piu chiaro e non grià giù oseuro dei settori. Cia non verificandosi, sembra probabile, whe le ridiazioni Rontgan si pieghino o Hettano dietro il disco, rischianandone in parte l'ombra, la quale pale s'oscure dallat perileriat al centro.

Risultati del tutto jdentici spno stati ottenuti tanto con un piccolo focus eliswoilale, che con uno serpico stiande.

lev contermare questa supposta flessione di ragrie determinarne possibilmente l'estensione l'A. si a serrito dell' ay-

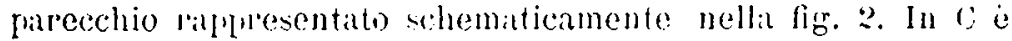

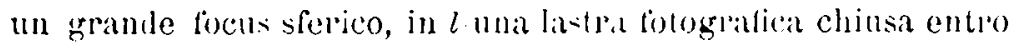
una scalola di cartone, posta in una cassia di piombo l' a grosse pareti. Per meglio apprezzare le differenze di toni sulla lastrat era stata posta una striscia di piombo. T'anto situando l'appa-

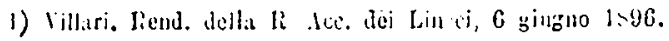




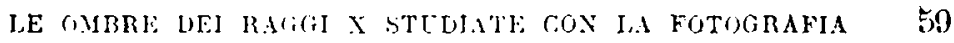

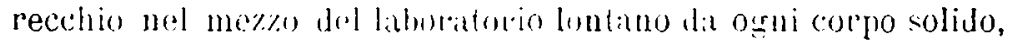

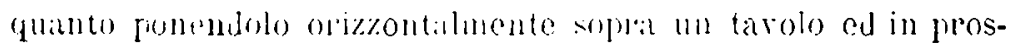

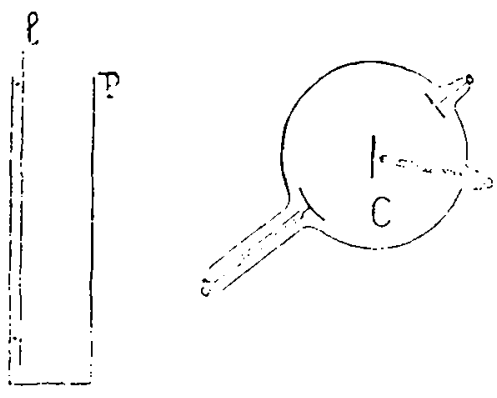

Fiะ. 2. simitil di llna prapete della stanzat, per alccrescere le sllposte e prosibili mflessumi degli X, i risultatiosselvati somn semple riusciti nli uni identici agli altri in tutti e due $i$ casi. Le futroglie ottenute con questu frocedimento sono simili a quelle arute adoperiundo il disco, si notano in exse le solite degradationi distinte che confermano la Heximos desli $\mathrm{x}$.

Se invere del focus s'adoperar un crookes a pera s'ottengrono delle fotosratie molto diverve. Yella fig. 3, qui appresso,

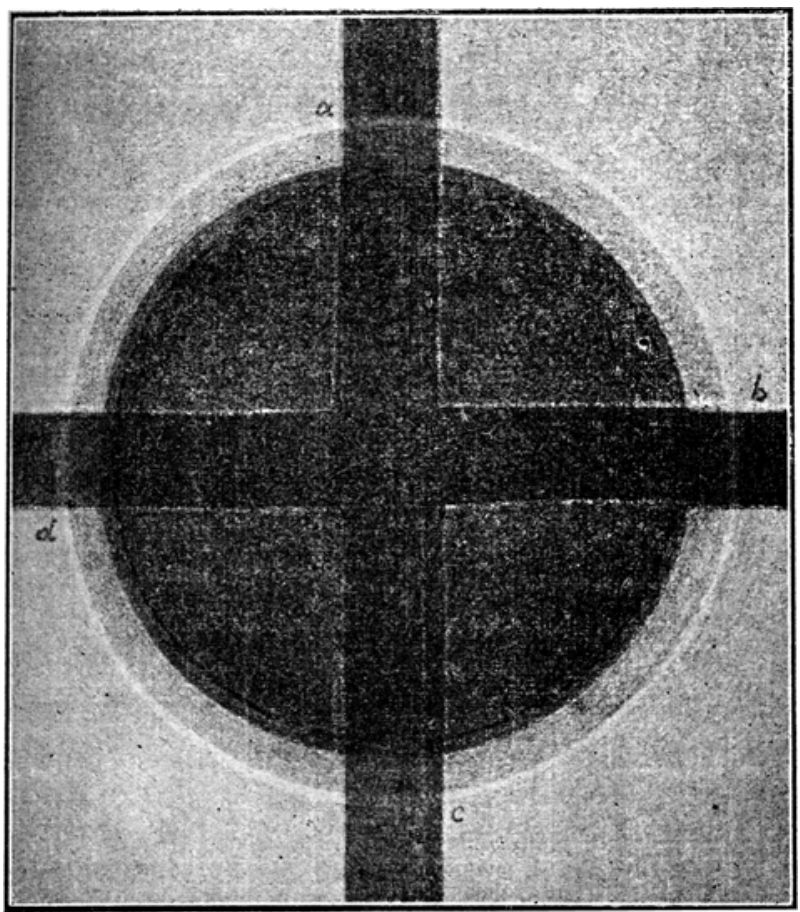

$\mathrm{Fi}_{\circ}$ :̈. 
che rappresenta lit positiva di una di coteste immagrini si scorge

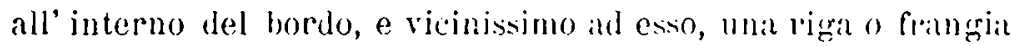
assai piu oscura del resto dell ombrib. Ia tinta dellat rigral degrada rapilannente all' interno, per prendere il tono dell' ombrib, e meno rapidiunente all' externo. I, oinbrat si termina allo esterno brtscamente ed a contorno notto; ed è seguita da una

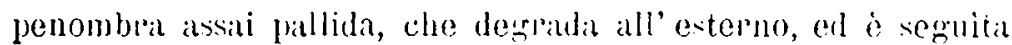

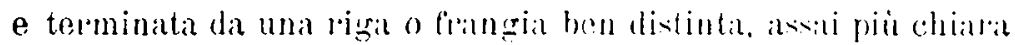

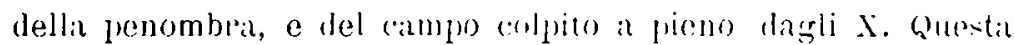

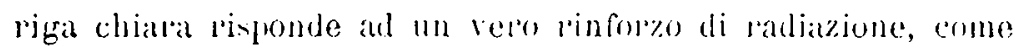
quella oscura ad mat diminuzione, fuasi fossero delle frange d'interlerenza.

In alcune esperienze, invece della croce di piombo ne adoperó una sottile di zinco, un po' tratspalente. Nolla positiva ottenuta, che e proprio quella riprodotta dellat lig. :3, le brar;cia della croce sono alquanto sbiadite, ed attraversate in $a, b$, $c$, a dalla frangia chialla, detta di sopra. Io che mostra, che le radiazioni, fungo la deta fiantia, fumono rinformate e trarersarono le blaccia della croce. Tel resto la fotografia è si inile a quella ottenutil col focus. Vi si regrono i settori, forse jiil chiari alla periferia cho al centro 1$)$, per una probabile ed estesa, ma debole flessione delle radiazioni.

L'A. fece anche uso di un crookes sferico, come quello della fig. 4, col catodo in c, aranti al quale fu posto il solito disco di piombo $a$ e la listrai fotograticial $l$. La fotografia ottenuta è illentica is quella prodota col croolies a perat, ed indicatia dalla figr. 3. Cosi che

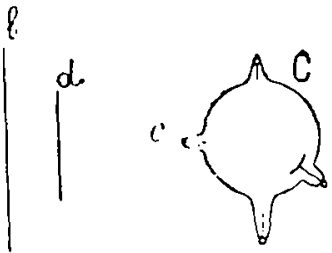

Fig. 4 . risulta, da fueste prove almeno, che i crookes dinno delle figure diverse dati focus.

I)a queste experienze l'A. hat tratto le seguenti conclusioni: $1^{\circ}$ Che l'ombra di un corpo che intercetta le radiazioni d'un focus è terminata da una fascia chiara, di parecchi mil.

1) La figura, per erroro, è piu chiturn al centro clo alla periferin: in realtit la croce pare si perda verso il centio perche rri j settori appariscono piu oscuri o piu neri. 
limelri, specie di panombari: ter.minata bruscamente all'esterno, e derriamata rapidamente verso il centro dell ombra.

$2^{n}$ Che l'ombra d' un corpo che intercetta, invece, lo radiarioni dun crookes, si termina con bordo netto e tagliente; pesso il bordo, nell' juterno dell ombra, scorgesi una riga o flatnoria nera: oltre il hordo osservasi mal penombla pallida, degradata all esterno, larogil parecohi millimetri, che é seguita da luna piga o trangia assili chiara, par pinforzo di radiazioni. Queste due foungre, la chiatra e la scura, ricordano quasi quelle di diffrazione.

$: 3^{0}$ L'ombra piona e centritle sombra cradatamente piu oscura dalla periferia al centro, per una probabile ed estesa flessione degli $x$ nell ombria greneratia la un coppo opaco che li intercettia.

L. MAGRI.

\section{LE SPIRALI CILINDRICHE DI FILO METALLICO COME MODELLO DELLE ONDE} STAZIONARIE LONGITODINALI,

del l'pof. (i. I3OX(iOONANi.

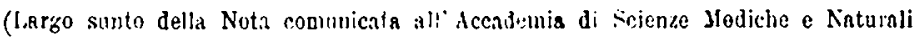
in Ferrara il gingno 24 Marzo 159s).
}

I.a relociti di froplatgrone delle ribmazioni longitudinali in un cilindro, solinlo, liquido o grazoso, e expressat dalla formulat :

$$
r=\sqrt{\bar{F}}
$$

nella quale E indica il modulo di elasticiti della sostanza e $d$ la suia densita.

Se indichiamo con $s$ lic sezione normale del cilindro, l'allungamento a dellia unitic di lunghezza del medesimo, quando esso viene sottoposto al una forma di trazione uguale al peso salg della sua uniti di lunghe\%a, $\iota^{2}$, per la formula notissima dell elaticitit di trazione:

$$
a=\frac{1}{\mathrm{l}} \cdot \mathrm{s} \cdot \mathrm{d} \cdot \mathrm{s}
$$

